\title{
Full Visible Range Covering InP/ZnS Nanocrystals with High Photometric Performance and Their Application to White Quantum Dot Light-Emitting Diodes
}

\author{
Xuyong Yang, Dewei Zhao, Kheng Swee Leck, Swee Tiam Tan, Yu Xin Tang, \\ Junliang Zhao, Hilmi Volkan Demir,* and Xiao Wei Sun*
}

Semiconductor nanocrystals (NCs), also known as colloidal quantum dots (QDs), exhibit unique size dependent properties and have attracted great interest thanks to their promising applications including light emitting diodes (LEDs), ${ }^{[1-8]}$ lasers, ${ }^{\left[{ }^{[9}-11\right]}$ biomedical imaging, ${ }^{[12,13]}$ and sensors. ${ }^{[14,15]}$ Currently, CdSe NCs as the workhorse have been well developed for such uses. ${ }^{[16-32]}$ Despite their apparent advantages (high emission quantum yields, narrow emission line width, good photostability, etc. ${ }^{[3-37]}$ ), the intrinsic toxicity of CdSe NCs makes them environmentally restricted, which has thus cast a doubtful future for their practical applications. ${ }^{[38,39]}$ Indium phosphide (band gap: $1.35 \mathrm{eV}$ ) is considered as the ideal alternative material, which offers a similar emission wavelength range but without intrinsic toxicity. ${ }^{[40-51]}$ Previous studies have demonstrated that InP NCs can be well used in white LEDs for improving their CRI. For example, Nann et al. reported a solidstate white LED with a CRI value up to 86 by adding InP/ZnS NCs in green together with yellow phosphors. ${ }^{[52]}$ Recently, a high quantum efficiency $(\mathrm{QE})$ of InP NCs of close to $70 \%$ was realized, ${ }^{[46]}$ and electroluminescence (EL) emission from InP-based NCs was also reported. ${ }^{[53]}$ However, there exist crucial problems related to the performance of these InP NCs. Among them are their color purity and emission spectrum tunability, which are much inferior to the well-developed CdSe NCs. The inferior properties of these InP NCs originate from a harsh reaction control owing to the strong coordinating strength of indium ligands. The emission linewidth of the typical InP NCs reported is wider (50-80 nm) than that of CdSe (15-40 nm), which leads to a worse color purity for InP as compared to CdSe. A pioneer work on the synthesis of the InP/ZnS NCs with tunable emission wavelength has been reported by Thomas Nann and co-workers. ${ }^{[54]}$ Although an impressively wide range emission spectrum tunability is achieved, the synthesis procedure used is quite complicated requiring continuous multiple injections for obtaining NCs with emission at longer wavelengths. As a result, it is still a great scientific challenge to obtain InP NCs with photometric performance as good as CdSe NCs.

In this communication, we report on the controlled synthesis of high-quality InP/ZnS core-shell NCs by a facile one-pot solvothermal method. The resulting NCs show high quantum yields (above $60 \%$ ), wide range spectrum tunability, narrow emission linewidths, and excellent photostability. The PL spectrum of the NCs is tuned to cover the entire visible spectrum by changing the ratio of InP:ZnS without multiple injections. The photoluminescence full width at half maxima (FWHM) of only $38 \mathrm{~nm}$ presented in this work is the narrowest linewidth reported for InP-based NCs to date. In addition, utilizing the resulting highquality InP/ZnS NCs, a white QD-LED has been demonstrated with a high color rendering index of 91 .

For a typical synthesis, indium myristate (In(MA)), tris(trimethylsilyl)phosphine $\left(\mathrm{P}(\mathrm{TMS})_{3}\right)$, and 1-octadecene (ODE) were mixed in glove box firstly (the color of solution quickly changes from colorless to yellow due to the nucleation process of InP). Next, the as-prepared InP nucleus solution was transferred into a three-neck flask, and then zinc stearate $\left(\mathrm{Zn}-\mathrm{St}_{2}\right)$ was added into the reaction system. The mixture was heated to $300{ }^{\circ} \mathrm{C}$ under Ar atmosphere and kept for a certain time to grow InP cores with Zn-rich surface. Subsequently, the precursor of $\mathrm{S}$ was injected into InP core-containing solution for growing $\mathrm{ZnS}$ shell. After that, an additional $\mathrm{ZnS}$ shell can
X. Yang, Dr. D. Zhao, K. S. Leck, Dr. S. T. Tan,

Prof. H. V. Demir, Prof. X. W. Sun

Luminous! Center of Excellence for Semiconductor

Lighting and Displays

School of Electrical and Electronic Engineering

Nanyang Technological University

Nanyang Avenue, Singapore 639798, Singapore

E-mail: hvdemir@ntu.edu.sg; EXWSun@ntu.edu.sg

Y. X. Tang

School of Materials Science and Engineering

Nanyang Technological University

Nanyang Avenue, Singapore 639798, Singapore

Prof. H. V. Demir

School of Physical and Mathematical Sciences

Nanyang Technological University

Nanyang Avenue, Singapore 639798, Singapore
Prof. H. V. Demir

Department of Electrical and Electronics Engineering Department of Physics

UNAM-Institute of Materials Science

and Nanotechnology

Bilkent University

Bilkent, Ankara, 06800, Turkey

Prof. J. L. Zhao

Department of Applied Physics

College of Science, and Tianjin Key Laboratory of Low-Dimensional

Functional Material Physics and Fabrication Technology

Tianjin University

Tianjin 300072, China

DOI: 10.1002/adma.201104990 


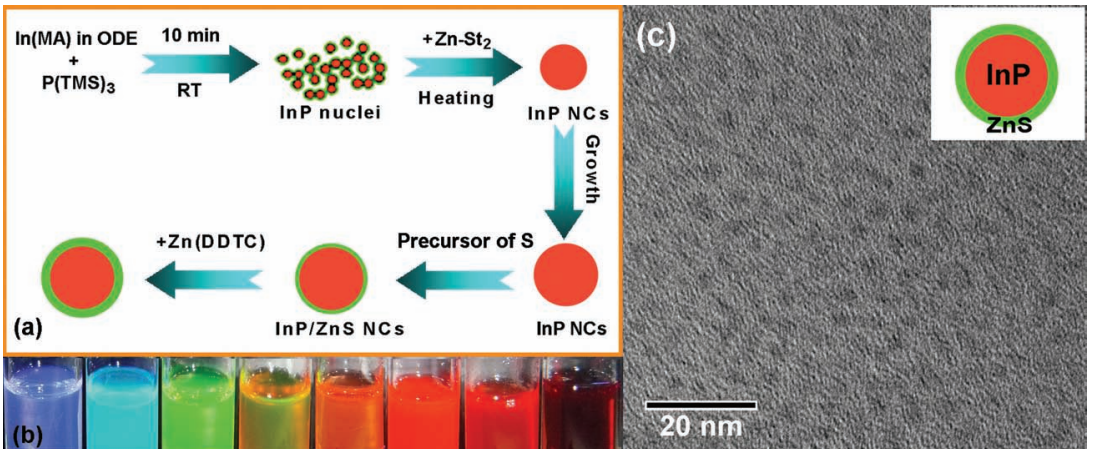

Figure 1. (a) Schematic of the synthetic procedure of $\ln P / Z n S$ NCs. (b) Photograph of the samples with different ratios of InP:ZnS excited by UV light. (c) TEM image of the resulting InP/ ZnS NCs at low magnification. The inset in (c) illustrates the core-shell structure of $\ln P / Z n S$ NCs. Here the red region represents InP core, and the green part show ZnS shell.
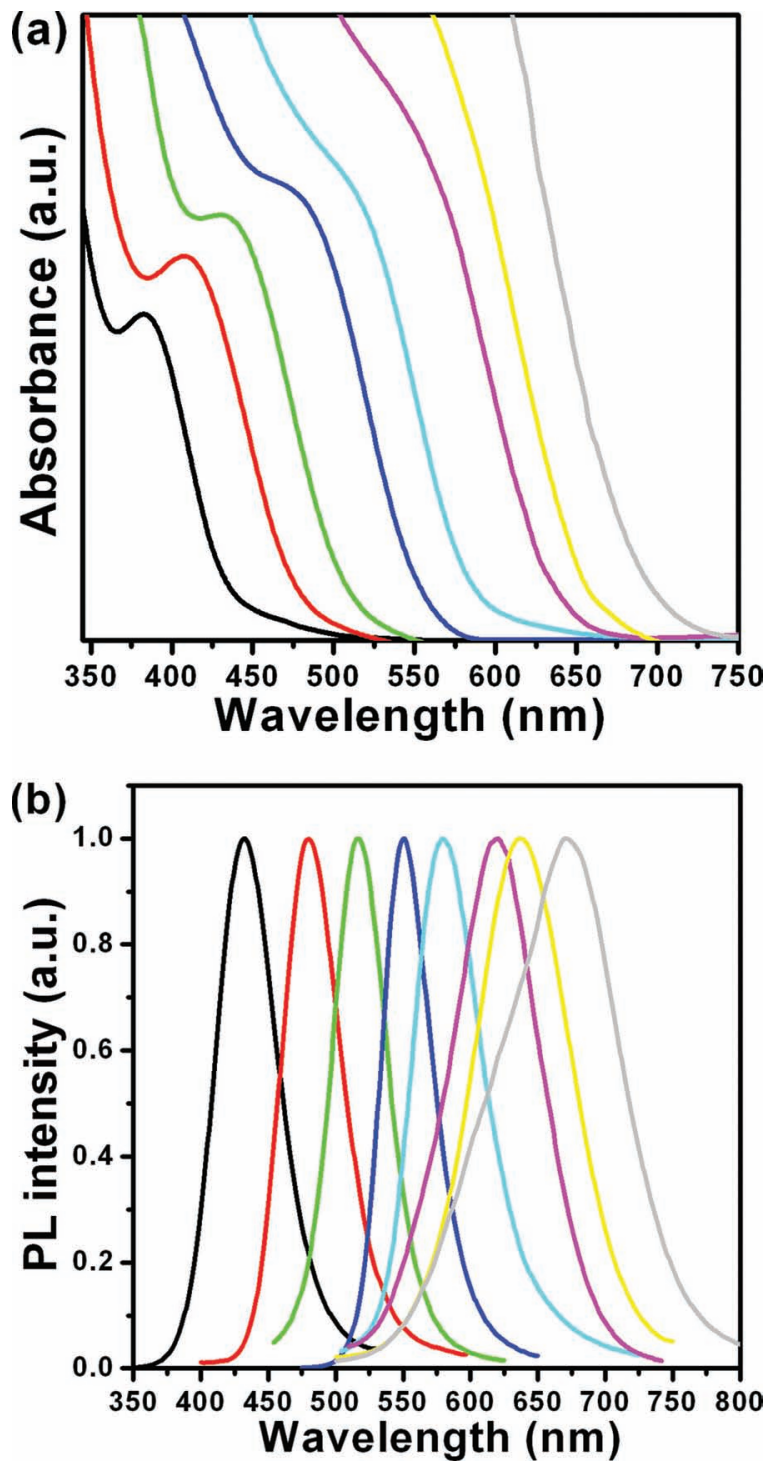

Figure 2. UV-Vis absorption and PL spectra of the resulting $\ln P / Z n S$ $\mathrm{NCs}$ with different ratios of InP:ZnS (increasing the ratio of InP to ZnS from left to right). The PL and absorption spectra of NCs were red-shifted successively. also be obtained by the decomposition of zinc diethyldithiocarbamate. The process is illustrated in Figure 1a. The resulting precipitate was dispersed in toluene. Energy-dispersive X-ray spectroscopy (EDS) confirmed that the resulting InP/ZnS NCs were composed by In, $\mathrm{P}, \mathrm{Zn}$, and $\mathrm{S}$ (Fig. S1, Supporting Information). The image of these NC samples with different ratios of InP:ZnS excited by UV light is shown in Figure 1b. The confirmation of a narrow size distribution can be directly observed by using a transmission electron microscope (TEM) (Figure 1c).

The UV-vis absorption and photoluminescence (PL) spectra of the as-grown InP/ $\mathrm{ZnS}$ NCs prepared at $300{ }^{\circ} \mathrm{C}$ are shown in Figure 2. We can see that both the absorption and PL spectra red-shift toward longer wavelengths with the increase of the molar ratio of InP:ZnS from 1:2 to 16:1. As previously reported, the addition of zinc carboxylates not only act as the zinc sources in the synthesis process of InP, but also influences the growth process of NCs by stabilizing the surfaces of particles and reduces the critical nucleus size. ${ }^{[51]}$ In our work, the absorption spectra and PL emission wavelength can be continuously tuned from blue to near-infrared (NIR) region by increasing the ratio of InP precursors to zinc stearate while keeping the other reaction conditions unchanged. Meanwhile, the zinc-rich surface of NCs facilitates the ZnS shell growth, after adding the precursor of $\mathrm{S}$, a homogeneous ZnS shell can be formed. The core-shell structure leads to an improved fluorescence quantum yield and a minor blue-shift of PL spectrum (Fig. S2, Supporting Information). It should be noted that the addition of zinc stearate cannot result in lattice doping because the bulk zinc doping of InP or preparation of $\mathrm{Zn}_{3} \mathrm{P}_{2}$ requires very harsh reaction conditions such as reactive zinc precursors, high temperature and a long reaction time. ${ }^{[46,51,54]}$

Figure 3 shows the fluorescence quantum yield (QY) and full width half maximum (FWHM) of the resulting InP/ZnS core-shell NCs. The high fluorescence QY and narrow emission

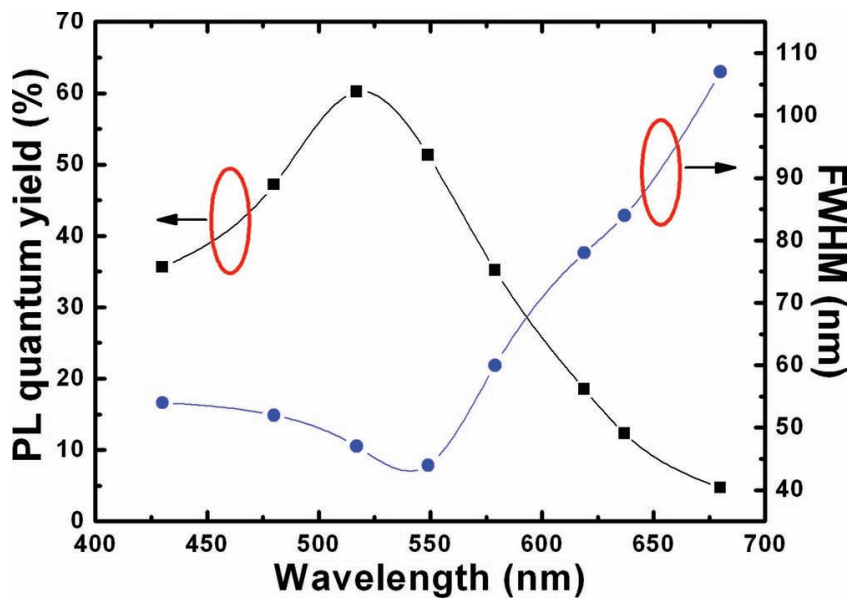

Figure 3. Evolution of fluorescence $Q Y$ and FWHM of $\ln P / Z n S N C s$ with different ratios of $\ln P: Z n S$ (increasing the ratio of $\operatorname{InP}$ to $\mathrm{ZnS}$ from left to right). 
linewidth can be obtained when the ratio of InP to $\mathrm{ZnS}$ is lower than $1.2: 1$, and the maximum QY is $60.2 \%$, which is comparable to that of CdSe NCs. The effective passivation of the surface-state traps of the NC cores in terms of the strain-relieved shells is considered as the origin of the observed high fluorescence QY. With the ratio of InP:ZnS further increasing, the fluorescence QY of NCs decreases gradually due to the decreased amount of $\mathrm{ZnS}$ that the thin $\mathrm{ZnS}$ shell cannot passivate the surface of InP cores well. Accordingly, the FWHM of NCs is also becoming wider due to the reduced amount of zinc stearate, which functions to stabilize the growth of NCs and results in a narrow particle size distribution.

The optical property of semiconductor NCs is strongly dependent on their surface ligands. It has been found that most ligands show stronger coordinating strength towards indium than cadmium. ${ }^{[54]}$ The presence of strong ligands for indium leads to unstable initial nuclei, and even particle growth at low temperature. Therefore, to reduce the negative influence of strong coordinating ligands, the formation of InP nuclei by mixing the In(MA) and $\mathrm{P}(\mathrm{TMS})_{3}$ in a non-coordinating solvent (octadecene in our case) was deliberately designed as a preliminary step in the nanocrystal synthesis (as evidenced by the color change of the reaction solution when the In(MA) and P(TMS $)_{3}$ were mixed). As shown in Figure 4a, the PL emission peak is shorter when the sample is prepared through the preliminary step in a non-coordinating solvent. It can be reasonably concluded that in this case the growth rate of NCs is slower, and the more stable synthesis process would favor the formation of NCs with uniform size.

Nevertheless, an excess of indium precursor in the preliminary reaction system was required to avoid the surface defects and aggregation of nanoparticles caused by the lack of surface ligands. The excess indium can provide an In-rich surface, which allows a sufficient amount of ligands to bind. It can be observed that the dangling indium bonds in the sample with In:P ratio of 1:1 result in a deep-trap surface emission at $630 \mathrm{~nm}$ in Figure 4b. In this case, defects at the NCs' surface can give rise to nonradiative recombination processes that lead to weak fluorescence QY ( 7.2\%). With the increase of In (In:P = 1.18:1), the defect emission disappears and the fluorescence QY is also enhanced significantly ( $50 \%)$. At the same time, the FWHM of the resulting NCs is remarkably narrowed (only $38 \mathrm{~nm}$ ), indicating the high color purity of NCs. Literature data correlating the FWHM of InP-based NCs is typically wider $(50-80 \mathrm{~nm})$ than that of CdSe $(15-40 \mathrm{~nm})$. The FWHM value of $38 \mathrm{~nm}$ reported in our work is very close to that of CdSe.

The ZnS shell plays a key role in improving the fluorescence QY of InP NCs. However, as the InP:ZnS ratio increases, the reduced amount of $\mathrm{ZnS}$ is too low to passivate the InP cores efficiently, resulting in a quick decrease of QY of NCs. Therefore, to solve the problem, we coat an additional $\mathrm{ZnS}$ shell on the initial InP/ZnS NCs with a thin ZnS shell by a one-step single source precursor injection. As shown in Figure 5a, the fluorescence QY of the initial red NCs is only $12.3 \%$, after growing an additional $\mathrm{ZnS}$ shell, the sample shows improved QY of $38.1 \%$.

Another serious negative influence on the resulting NCs caused by the thin $\mathrm{ZnS}$ shell is the poor photostability. The ZnS shell would become very thin as the amount of $\mathrm{Zn}$ precursor
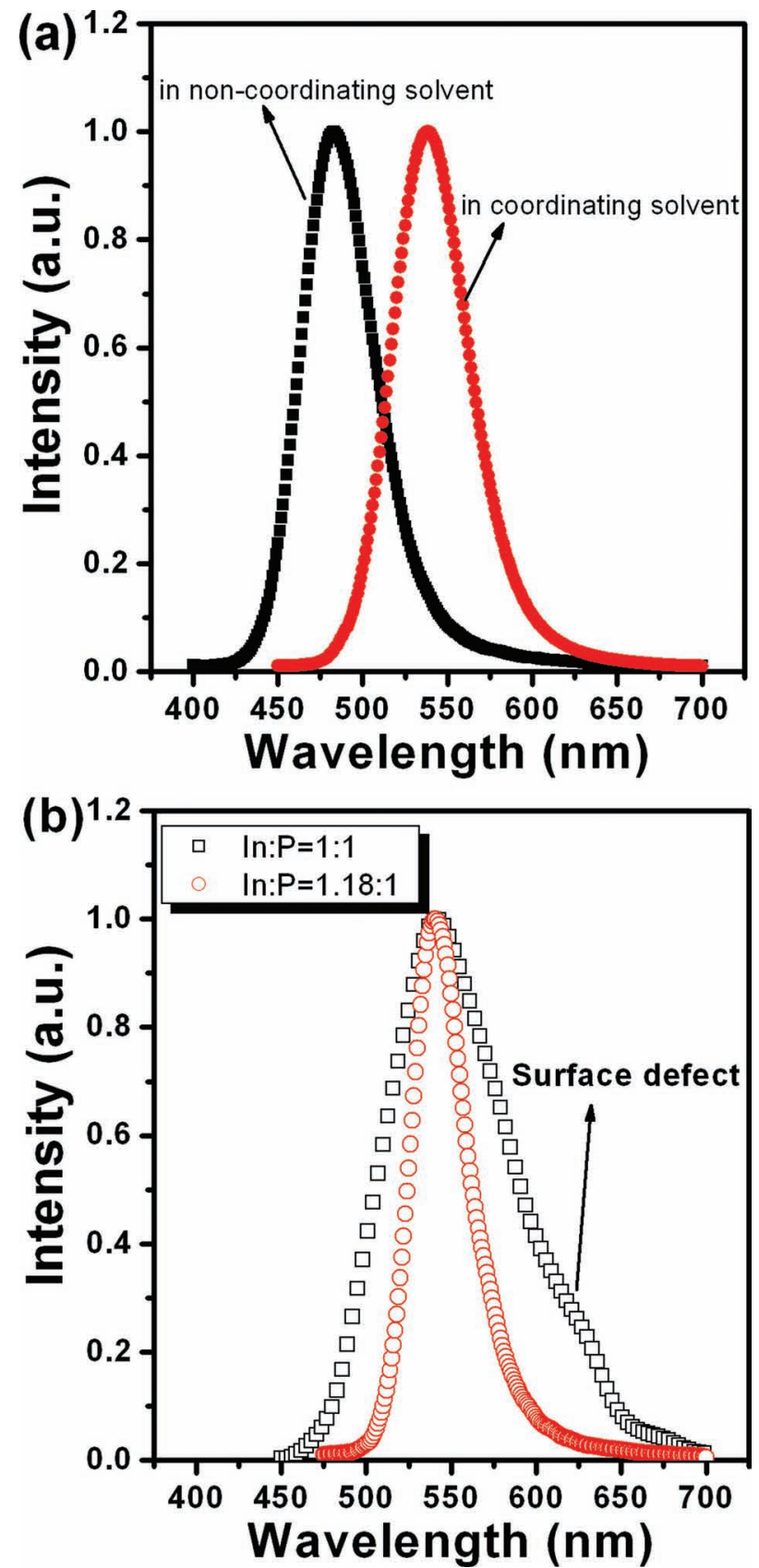

Figure 4. PL spectra of (a) the resulting $\ln P / Z n S N C s$ prepared through a preliminary step (black dots) and without preliminary step (red dots) and (b) the samples prepared with different ratios of In:P.

used reduces constantly. Such a thin shell is very vulnerable against degradable conditions, causing dramatic reduction in the photostability. This could be assessed by continuous irradiation with UV light. As shown in Figure 5b, for the NCs without additional ZnS shell, a rapid decrease of their PL intensity (which decreases to $70 \%$ of its initial value) can be observed within $10 \mathrm{~h}$ of UV irradiation $\left(365 \mathrm{~nm}, 3 \mathrm{~mW} / \mathrm{cm}^{2}\right)$. On the contrary, for the NCs with an additional ZnS shell, even after $25 \mathrm{~h}$ of continuous irradiation, little photodegradation can be seen. 

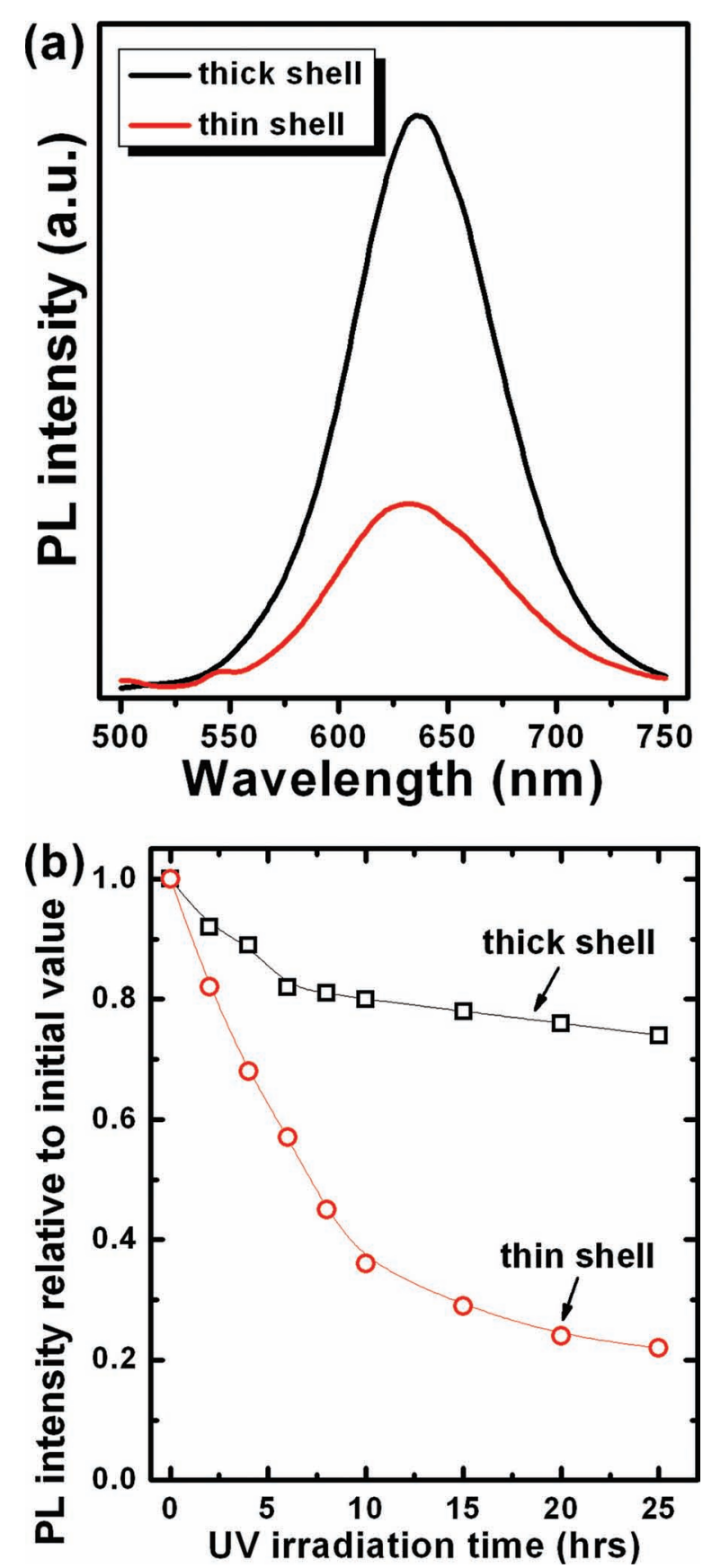

Figure 5. (a) PL intensity and (b) photostability of the resulting $\ln P / Z n S$ NCs with additional ZnS shell and without additional ZnS shell.

To assess suitability of the resulting InP/ZnS core-shell NCs as emitters for QD-LED application, a device structure of ITO/ PEDOT:PSS/poly-TPD/QDs/TPBi/LiF/Al was fabricated, for which the corresponding schematic energy level diagram is shown in Figure 6a. The LED output spectrum is recorded at the bias voltage of $11 \mathrm{~V}$ (Figure $6 \mathrm{~b}$ ), exhibiting a broadband coverage over the entire visible wavelength regime. The red emission is attributed to the red-emitting InP/ZnS NCs (confirmed by the PL profile of red NCs), which is spectrally complementary to the blue-green emission of poly-TPD for white light generation. The CIE coordinates of the cadmium-free LED is $(0.332$, 0.338 ), which is close to the balanced white coordinates (inset of Figure 6c). In addition, the white light of the QD-LED is characterized with a high CRI of 91, significantly higher than that of most of solid-state white LEDs. An image of the white QD-LED output was recorded at a luminance of $270 \mathrm{~cd} / \mathrm{m}^{2}$ (inset of Figure 6c), which displays bright and uniform white emission.

In summary, high-quality InP/ZnS core-shell NCs with luminescence tunable over the entire visible spectrum have been prepared by a facile one-pot solvothermal method without multiple injections. The excellent optical properties of the resulting NCs including high quantum yields, narrow emission linewidth and good photostability were obtained by effectively passivating the surface defects of InP NC cores with a strain-relieved $\mathrm{ZnS}$ shell. The FWHM of the NCs is as narrow as $38 \mathrm{~nm}$. Furthermore, in this work, a white QD-LED based on the resulting NCs has been successfully demonstrated with a high color rendering index of 91. The high performance of the resulting InP/ $\mathrm{ZnS}$ NCs coupled with their low intrinsic toxicity may further enable industrial applications of these NC emitters.

\section{Experimental Details}

Materials: indium acetate (99.99\%, Alfa Aesar), myristic acid ( $\geq 99 \%$, Sigma), tris(trimethylsilyl) phosphine $\left(\mathrm{P}(\mathrm{TMS})_{3} ; 95 \%\right.$, Aldrich), zinc stearate (10-12\% Zn basis, Sigma-Aldrich), zinc diethyldithiocarbamate (Zn(DDTC) $2 ; 97 \%$, Aldrich), Sulfur powder (S; $\geq 99.5 \%$, Sigma-Aldrich), 1-octadecene (ODE; 90\%, Aldrich), 1-dodecanethiol (DDT; 98\%, Alfa Aesar), ethanol (AR), chloroform (AR), toluene (AR). All chemicals were used as received without further purification. The InP-ZnS core-shell nanocrystals were synthesized by using standard air-free procedures.

Synthesis of monodisperse InP/ZnS core-shell nanocrystals: A typical procedure is given as follows: $0.3 \mathrm{mmol}$ of $\ln (\mathrm{MA})_{3}, 0.3 \mathrm{mmol}$ of $\mathrm{P}(\mathrm{TMS})_{3}$ and $3 \mathrm{~mL}$ of ODE were fully mixed in a nitrogen glove box at room temperature (following which the color of solution quickly change from colorless to yellow due to the nucleation process of InP). Next, the as-prepared InP nuclei solution was transferred into a three-neck flask $(50 \mathrm{~mL}$ ), and then $0.3 \mathrm{mmol}$ of zinc stearate and $21 \mathrm{~mL}$ of ODE were added into the reaction system. The mixture was quickly heated to $260-300{ }^{\circ} \mathrm{C}$ with strong agitation under $\mathrm{Ar}$ atmosphere and kept at this temperature for a defined time $(10-30 \mathrm{~min})$ to grow $\ln P$ cores. Subsequently, $0.3 \mathrm{mmol}$ of DDT or S powder was added into $\operatorname{InP}$ corecontaining solution which was cooled down to $60^{\circ} \mathrm{C}$ around beforehand, and was repeatedly heated up to $300{ }^{\circ} \mathrm{C}$ for $10 \mathrm{~min}$. After cooled down to room temperature, $54 \mathrm{mg}$ of zinc diethyldithiocarbamate and $3 \mathrm{~mL}$ of ODE was added to the solution for growing an additional $\mathrm{ZnS}$ shell. The flask was heated to $220-230^{\circ} \mathrm{C}$ again for $20 \mathrm{~min}$. To purify the resulting $\mathrm{NCs}$, the reaction mixture was cooled down to room temperature, and $3 \mathrm{~mL}$ of a chloroform/ethanol (2:1 vol:vol) mixture as well as an excess amount of acetone were added, followed by centrifugation. The as-prepared precipitate could readily be dispersed in toluene or chloroform. The precursor indium myristate was prepared using the method introduced in Ref. 46.

Fabrication of the QD-LED device: The patterned ITO substrates were cleaned by sonication sequentially in detergent, de-ionized water acetone, and isopropyl alcohol. The PEDOT:PSS was spun on the plasma$\mathrm{O}_{2}$-treated ITO substrate at a spin rate of $4000 \mathrm{rpm}$ for $60 \mathrm{~s}$ and dried at $150{ }^{\circ} \mathrm{C}$ for $30 \mathrm{~min}$ in a nitrogen glove box. The $1 \mathrm{wt} \%$ of poly-TPD in chlorobenzene was also spin-coated on the PEDOT:PSS layer at $2000 \mathrm{rpm}$ for $60 \mathrm{~s}$, followed by thermal annealing at $110^{\circ} \mathrm{C}$ for $30 \mathrm{~min}$. The QD layer was then deposited on the ITO/PEDOT:PSS/poly-TPD layer 
(a)

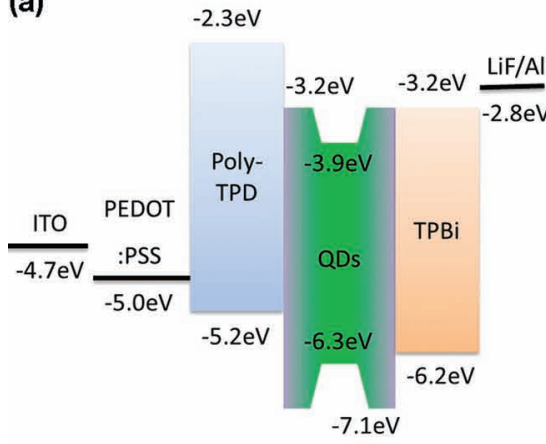

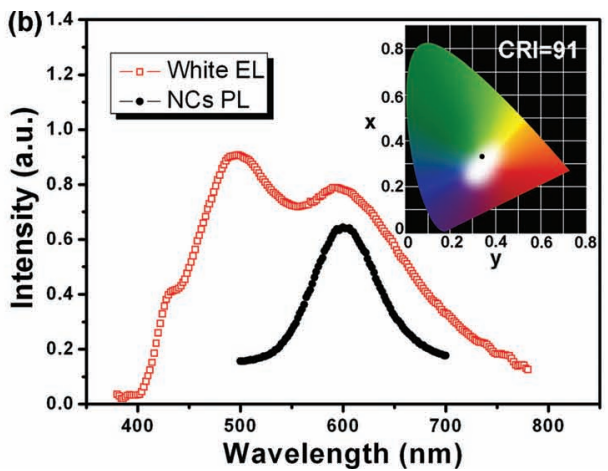

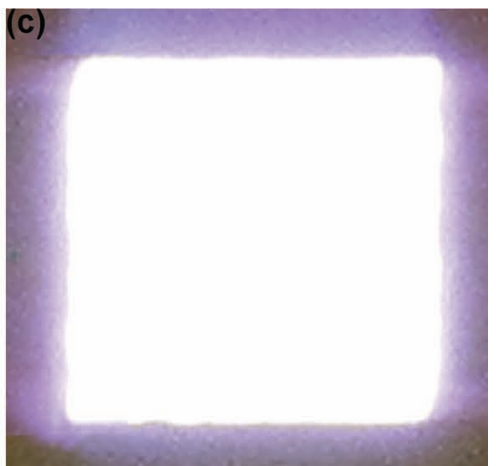

Figure 6. (a) Energy band diagram of the QD-LEDs using InP/ZnS QDs. The theoretical values for the valence (or HOMO) and conduction bands (or LUMO) for individual layers were obtained from Ref 32. (b) PL spectrum of red-emitting InP/ZnS NCs and EL spectrum of white QD-LEDs. (c) CIE chromaticity coordinate diagram of the white QD-LEDs and a photograph of a QD-LED with a pixel size of $3 \mathrm{~mm} \times 3 \mathrm{~mm}$ (inset).

by spin-coating the QD dispersion (InP/ZnS NCs dispersed in toluene with $10 \mathrm{mg} / \mathrm{mL}$ ) at a rate of $1000-2000 \mathrm{rpm}$ for $60 \mathrm{~s}$, and cured at $60^{\circ} \mathrm{C}$ under $\mathrm{N}_{2}$ atmosphere for $30 \mathrm{~min}$. The TPBi $(35 \mathrm{~nm})$, LiF $(0.5 \mathrm{~nm})$, and Al $(150 \mathrm{~nm})$ layers were thermally deposited under a based pressure of $\sim 2 \times 10^{-4} \mathrm{~Pa}$.

Instrumentation: Transmission electron microscopy (TEM) characterization was performed with a transmission electron microscope (JEOL, JEM-2010) operating at $200 \mathrm{kV}$. Samples for TEM analysis were prepared by drying a drop of nanocrystals dispersion in toluene on amorphous carbon-coated copper grids. The Energydispersive X-ray spectroscopy (EDS) was obtained using a field emission scanning electronic microscope (FESEM, JEOL JSM-6340F). The photoluminescence $(\mathrm{PL})$ spectra were recorded in the spectral range of $350-800 \mathrm{~nm}$ at room temperature (Fluorolog-3 Spectrofluorometer). The absorption spectra were obtained over a wavelength range from 350 to $750 \mathrm{~nm}$ using a UV-vis spectrophotometer (Shimadzu) with a $50 \mathrm{~W}$ halogen lamp and a deuterium lamp as the excitation source. The fluorescence QY of InP/ZnS nanocrystals was measured by comparing the integrated area of photoluminescence emission of rhodamine 6C in ethanol (QY $=95 \%)$ with $\operatorname{lnP} / \mathrm{ZnS}$ nanocrystals in toluene, with the same absorbance value at excitation wavelength and similar fluorescence wavelength. The electroluminescence (EL) spectra of the fabricated devices were measured using a PR650 Spectra Scan spectrometer, while the luminance-current density-voltage (L-J-V) characteristics were obtained simultaneously, by connecting the spectrometer to a programmable Keithley 236 source measurement unit. All measurements were carried out at room temperature under ambient conditions.

\section{Supporting Information}

Supporting Information is available from the Wiley Online Library or from the author.

\section{Acknowledgements}

The authors would like to thank the financial support from Singapore National Research Foundation (NRF) under NRF-RF-2009-09, NRF-CRP-2011-02 and the Science and Engineering Research Council, Agency for Science, Technology and Research (A*STAR) of Singapore (project No. 092101 0057). The work is also supported by the National Natural Science Foundation of China (NSFC) (project Nos. 61006037 and 61076015).

Received: December 31, 2011 Published online: April 30, 2012
[1] L. Qian, Y. Zheng, J. Xue, P. H. Holloway, Nature Photon. 2011, 5, 543.

[2] H. V. Demir, S. Nizamoglu, T. Erdem, E. Mutlugun, N. Gaponikc, A. Eychmüller, Nano Today 20116, 632.

[3] D. P. Puzzo, E. J. Henderson, M. G. Helander, Z. Wang, G. A. Ozin, Z. Lu, Nano Lett. 2011, 11, 1585.

[4] Z. Tan, Y. Zhang, C. Xie, H. Su, J. Liu, C. Zhang, N. Dellas, S. E. Mohney, Y. Wang, J. Wang, J. Xu, Adv. Mater. 2011, 23, 3553.

[5] E. M. Likovich, R. Jaramillo, K. J. Russell, S. Ramanathan, V. Narayanamurti, Adv. Mater. 2011, 23, 4521.

[6] E. Jang, S. Jun, H. Jang, J. Lim, B. Kim, Y. Kim, Adv. Mater. 2010, 22, 3076.

[7] H. S. Jang, H. Yang, S. W. Kim, J. Y. Han, S. G. Lee, D. Y. Jeon, Adv. Mater. 2008, 9999, 1.

[8] J. W. Stouwdam, R. A. J. Janssen, Adv. Mater. 2009, 21, 2916.

[9] A. V. Malko, Y. S. Park, S. Sampat, C. Galland, J. Vela, Y. Chen, J. A. Hollingsworth, V. I. Klimov, H. Htoon, Nano Lett. 2011, 11, 5213.

[10] E. R. Smith, J. M. Luther, J. C. Johnson, Nano Lett. 2011, 11, 4923.

[11] J. Wang, M. Lin, Y. Yan, Z. Wang, P. C. Ho, K. P. Loh, J. Am. Chem. Soc. 2009, 131, 11300.

[12] L. M. Maestro, E. M. Rodríguez, F. S. Rodríguez, M. C. Iglesias-de la Cruz, A. Juarranz, R. Naccache, F. Vetrone, D. Jaque, J. A. Capobianco, J. G. Solé, Nano Lett. 2010, 10, 5109.

[13] P. M. Allen, W. Liu, V. P. Chauhan, J. Lee, A. Y. Ting, D. Fukumura, R. K. Jain, M. G. Bawendi, J. Am. Chem. Soc. 2010, 132, 470.

[14] N. E. Coates, H. Zhou, S. Krämer, L. Li, D. Moses, Adv. Mater. 2010, 5366, 22, 5366.

[15] S. Miao, S. G. Hickey, B. Rellinghaus, C. Waurisch, A. Eychmüller, J. Am. Chem. Soc. 2010, 132, 5613.

[16] K. S. Cho, E. K. Lee, W. J. Joo, E. Jang, T. H. Kim1, S. J. Lee, S. J. Kwon, J. Y. Han, B. K. Kim, B. L. Choi1, J. M. Kim, Nature Photon. 2009, 3, 341.

[17] J. M. Caruge, J. E. Halpert, V. Wood, V. Bulović, M. G. Bawendi, Nature Photon. 2008, 2, 247.

[18] Q. Sun, Y. A. Wang, L. S. Li, D. Wang, T. Zhu, J. Xu, C. Yang, Y. Li, Nature Photon. 2007, 1, 717.

[19] Y. Zhang, C. Xie, H. Su, J. Liu, S. Pickering, Y. Wang, W. W. Yu, J. Wang, Y. Wang, J. I. Hahm, N. Dellas, S. E. Mohney, J. Xu, Nano Lett. 2011, 11, 329.

[20] W. K. Bae, J. Kwak, J. Lim, D. Lee, M. K. Nam, K. Char, C. Lee, S. Lee, Nano Lett. 2010, 10, 2368.

[21] M. J. Panzer, K. E. Aidala, P. O. Anikeeva, J. E. Halpert, M. G. Bawendi, V. Bulović, Nano Lett. 2010, 10, 2421.

[22] M. A. Schreuder, K. Xiao, I. N. Ivanov, S. M. Weiss, S. J. Rosenthal, Nano Lett. 2010, 10, 573 
[23] V. Wood, M. J. Panzer, J. M. Caruge, J. E. Halpert, M. G. Bawendi, V. Bulović, Nano Lett. 2010, 10, 24.

[24] L. Kim, P. O. Anikeeva, S. A. Coe-Sullivan, J. S. Steckel, M. G. Bawendi, V. Bulović, Nano Lett. 2008, 8, 4513.

[25] P. O. Anikeeva, J. E. Halpert, M. G. Bawendi, V. Bulović, Nano Lett. 2009, 9, 2532

[26] Z. Tan, F. Zhang, T. Zhu, J. Xu, A. Y. Wang, J. D. Dixon, L. Li, Q. Zhang, S. E. Mohney, Nano Lett. 2007, 7, 3803.

[27] P. O. Anikeeva, J. E. Halpert, M. G. Bawendi, V. Bulović, Nano Lett. 2007, 7, 2196.

[28] J. Zhao, J. A. Bardecker, A. M. Munro, M. S. Liu, Y. Niu, I. K. Ding, J. Luo, B. Chen, A. K. Y. Jen, D. S. Ginger, Nano Lett. 2006, 6, 463.

[29] T. Nann, W. M. Skinner, ACS Nano 2011, 5, 5291.

[30] V. Wood, M. J. Panzer, J. E. Halpert, J. M. Caruge, M. G. Bawendi, V. Bulović, ACS Nano 2009, 3, 3581.

[31] M. Zorn, W. K. Bae, J. Kwak, H. Lee, C. Lee, R. Zentel, K. Char, ACS Nano 2009, 3, 1063.

[32] Q. Sun, G. Subramanyam, L. Dai, M. Check, A. Campbell, R. Naik, J. Grote, Y. Wang, ACS Nano 2009, 3, 737.

[33] E. Talgorn, M. A. de Vries, L. D. A. Siebbeles, A. J. Houtepen, ACS Nano, 2011, 5, 3552.

[34] A. K. Gooding, D. E. Gómez, P. Mulvaney, ACS Nano 2008, 2, 669

[35] W. K. Bae, K. Char, H. Hur, S. Lee, Chem. Mater. 2008, 20, 531.

[36] B. Blackman, D. M. Battaglia, T. D. Mishima, M. B. Johnson, X. Peng, Chem. Mater. 2007, 19, 3815.

[37] N. Pradhan, D. Goorskey, J. Thessing, X. Peng, J. Am. Chem. Soc. 2005, 127, 17586
[38] T. Erdem, H. V. Demir, Nat. Photon. 2011, 5, 126.

[39] D. V. Talapin, J. S. Lee, M. V. Kovalenko, E. V. Shevchenko, Chem. Rev. 2010, 110, 389

[40] P. M. Allen, B. J. Walker, M. G. Bawendi, Angew. Chem. 2010, 122 772

[41] Z. Liu, A. Kumbhar, D. Xu, J. Zhang, Z. Sun, J. Fang, Angew. Chem. 2008, 120,3596.

[42] S. Kim, J. Park, T. Kim, E. Jang, S. Jun, H. Jang, B. Kim, S. W. Kim, Small 2011, 7, 70

[43] H. Chibli, L. Carlini, S. Park, N. M. Dimitrijevic, J. L. Nadeau, Nanoscale 2011, 3, 2552

[44] D. D. Lovingood, G. F. Strouse, Nano Lett. 2008, 8, 3394.

[45] R. Xie, X. Peng, J. Am. Chem. Soc. 2009, 131, 10645.

[46] L. Li, P. Reiss, J. Am. Chem. Soc. 2008, 130, 11588.

[47] R. Xie, D. Battaglia, X. Peng, J. Am. Chem. Soc. 2007, 129, 15432.

[48] S. Tamang, G. Beaune, I. Texier, P. Reiss, ACS Nano 2011, 5, 9392.

[49] K. Huang, R. Demadrille, M. G. Silly, F. Sirotti, P. Reiss, O. Renault, ACS Nano 2010, 4, 4799.

[50] A. Narayanaswamy, L. F. Feiner, A. Meijerink, P. J. van der Zaag, ACS Nano 2009, 3, 2539.

[51] E. Ryu, S. Kim, E. Jang, S. Jun, H. Jang, B. Kim, S. W. Kim, Chem. Mater. 2009, 21, 573

[52] J. Ziegler, S. Xu, E. Kucur, F. Meister, M. Batentschuk, F. Gindele, T. Nann, Adv. Mater. 2008, 20, 4068.

[53] J. Lim, W. K. Bae, D. Lee, M. K. Nam, J. Jung, C. Lee, K. Char, S. Lee, Chem. Mater. 2011, 23, 4459.

[54] S. Xu, J. Ziegler, T. Nann, J. Mater. Chem. 2008, 18, 2653. 